\title{
Investigating neurovascular coupling using canonical correlation analysis between pharmacological MRI and electrophysiology Felix Bießmann*1,2, Arthur Gretton ${ }^{2}$, Frank C Meinecke ${ }^{1}$, Xiaoxhe Zhang2, Gregor Rainer ${ }^{2}$, Nikos K Logothetis ${ }^{2}$, Klaus-Robert Müller ${ }^{1}$ and
} Alexander Rauch ${ }^{2}$

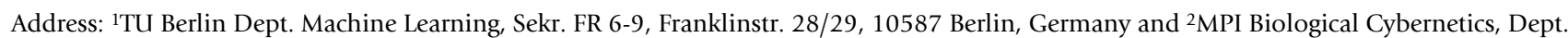 \\ Neurophysiology, Spemannstr. 41, 72076 Tübingen, Germany \\ Email: Felix Bießmann* - fbiessma@tuebingen.mpg.de \\ * Corresponding author
}

from Eighteenth Annual Computational Neuroscience Meeting: CNS*2009

Berlin, Germany. 18-23 July 2009

Published: 13 July 2009

BMC Neuroscience 2009, I0(SuppI I):P86 doi:10.1 I86/I47I-2202-I0-SI-P86

This abstract is available from: http://www.biomedcentral.com/I47I-2202/I0/SI/P86

(c) 2009 Bießmann et al; licensee BioMed Central Ltd.

\section{Poster presentation}

Despite its young age, functional Magnetic Resonance Imaging (fMRI) has become one of the most popular brain imaging techniques. However, the relationship between brain activity and the blood oxygen level dependent (BOLD) contrast as measured with fMRI, the so called neurovascular coupling, is not yet fully understood. One possibility of experimentally manipulating the neurovascular coupling mechanisms is administration of vaso-active and neuro-active substances, such as Acetylcholine (ACh). Combining those pharmacological interventions with simultaneous measurements of electrophysiological and BOLD response allows for deeper insights in the dependencies between neural and hemodynamic response to sensory stimulation.

We developed a method based on kernel canonical correlation analysis that is able to deal with the high dimensionality of the fMRI signal while exploiting the high temporal resolution of the electrophysiology. The algorithm finds filters for fMRI and electrophysiological data that maximize the crosscorrelation between the two data sources. Projecting the data onto those filters allows to compute a crosscorrelation function between fMRI and electrophysiological data that reflects the coupling between neural and hemodynamic response. We present data recorded in primary visual cortex of the non-human primate during a visual stimulation paradigm and local application of ACh. Comparing the neurovascular crosscorrellograms after local injections of ACh and with those from control conditions, we find that the coupling is dramatically affected by ACh. In particular, the extent to which the stimulus is reflected in the crosscorrelation function is decreased under influence of ACh. Inspection of the spatial filters of the BOLD response shows that this change is primarily accounted for by cholinergic effects on voxels around the injection site. The filters computed for the neurophysiological data suggest that it is mainly neural activity in the alpha and gamma band that contributes to the change in coupling.

In summary, the results provide preliminary evidence for a change in neurovascular coupling induced by high levels of ACh. The voxel patterns (for fMRI data) and patterns in the time-frequency domain (for electrophysiological data) that give rise to this change can be revealed using a novel analysis method. 